OPEN ACCESS

Edited by:

Qi Zhao,

Liaoning University, China

Reviewed by:

Patrick R. M. Harnarayan,

The University of the West Indies at St. Augustine, Trinidad and Tobago

Maria José Saavedra,

Universidade de Trás-os-Montes e Alto Douro, Portugal

*Correspondence: Honghua Hu

helen.hu@mq.edu.au

Specialty section:

This article was submitted to Systems Microbiology, a section of the journal Frontiers in Microbiology

Received: 22 May 2020 Accepted: 29 June 2020

Published: 22 July 2020

Citation:

Heravi FS, Zakrzewski M, Vickery K, Malone M and Hu H (2020) Metatranscriptomic Analysis Reveals Active Bacterial Communities in Diabetic Foot Infections.

Front. Microbiol. 11:1688. doi: 10.3389/fmicb.2020.01688

\section{Metatranscriptomic Analysis Reveals Active Bacterial Communities in Diabetic Foot Infections}

\author{
Fatemah Sadeghpour Heravi', Martha Zakrzewski², Karen Vickery', Matthew Malone 3,4,5 \\ and Honghua $\mathrm{Hu}^{1 *}$ \\ ${ }^{1}$ Surgical Infection Research Group, Faculty of Medicine and Health Sciences, Macquarie University, Sydney, NSW, \\ Australia, ${ }^{2}$ QIMR Berghofer Medical Research Institute, Brisbane, QLD, Australia, ${ }^{3}$ Infectious Diseases and Microbiology, \\ School of Medicine, Western Sydney University, Sydney, NSW, Australia, ${ }^{4}$ Liverpool Hospital, South Western Sydney LHD, \\ Sydney, NSW, Australia, ${ }^{5}$ Liverpool Diabetes Collaborative Research Unit, Ingham Institute for Applied Medical Research, \\ Sydney, NSW, Australia
}

Despite the extended view of the composition of diabetic foot infections (DFIs), little is known about which transcriptionally active bacterial communities are pertinent to infection, and if any differences are associated with increased infection severity. We applied a RNA sequencing approach to analyze the composition, function, and pathogenicity of the active bacterial communities in DFIs. Taxonomic profiling of bacterial transcripts revealed the presence of 14 bacterial phyla in DFls. The abundance of the Spiroplasma, Vibrio, and Mycoplasma were significantly different in different infection severities $(P<0.05)$. Mild and severe stages of infections were dominated by Staphylococcus aureus and Porphyromonas asaccharolytica, respectively. A total of 132 metabolic pathways were identified of which ribosome and thiamin being among the most highly transcribed pathways. Moreover, a total of 131 antibiotic resistance genes, primarily involved in the multidrug efflux pumps/exporters, were identified. Furthermore, iron acquisition systems (synthesize and regulation of siderophores) and pathways involved in the synthesis and regulation of cell-surface components associated with adhesion, colonization, and movement of bacterial cells were the most common virulence factors. These virulence factors may help bacteria compete for scares resources and survive the host wound proteases. Characterization of transcriptionally active bacterial communities can help to provide an understanding of the role of key pathogens in the development of DFls. Such information can be clinically useful allowing replacement of DFls empirical therapy with targeted treatment.

Keywords: diabetic foot infection, RNA sequencing, metatranscriptomics, active bacterial community, resistome, virulence factors

\section{INTRODUCTION}

Diabetic foot infections (DFIs) are a frequent cause of hospitalization and typically precede events such as lower extremity amputation (Lavery et al., 2003). Traditional approaches to identify pathogens colonized in DFIs have relied on culture-based methods that are limited to detect bacterial species grown under standard laboratory conditions (Heravi et al., 2020). 
Over the last decade, several studies have identified that diabetic foot ulcers were composed of a complex bacterial community consisting of aerobes, anaerobes, fastidious, and unculturable microorganisms using 16S rRNA sequencing (Smith et al., 2016; Gardiner et al., 2017). The 16S rRNA approach is limited to genomically present but transcriptionally inactive bacterial communities. It also does not provide insight into the potential or actual function of the bacterial community in DFIs.

High-throughput RNA sequencing or metatranscriptomic analysis is a promising tool to obtain insights into the functionality of active bacterial communities. Metatranscriptomics has also addressed the limitation of microarray assays such as unspecific hybridization signals (Zhao et al., 2016).

The composition and function of the active bacterial communities in DFIs can provide a clue for understanding the actual role of microorganisms in infection progression and the improvement of therapeutic approaches. To the best of our knowledge, this is the first study applying an RNA sequencing approach to explore the composition, function, and virulence factors of the transcriptionally active bacteria in DFIs.

\section{MATERIALS AND METHODS}

\section{Ethics Statement}

This study was approved by the South West Sydney Local Health District Research and Ethics Committee (HREC/14/LPOOL/487, SSA/14/LPOOL/489) and Macquarie University Human Ethics Committee (Reference No. 5201500839). All the experiments were performed in accordance with relevant guidelines and regulations. Informed consent has been obtained for this study.

\section{Patient Population}

In this prospective study, 43 consecutive patients aged over 18 years presenting to the Liverpool Hospital High-Risk Foot Service with a clinically diagnosed DFI were enrolled over a 6-month period. Infection severity was determined using the International Working Group of the Diabetic Foot (IWGDF), Perfusion, Extent, Depth, Infection and Sensation (PEDIS) classification system, and patients were assigned accordingly (PEDIS 2 refers to mild infection, PEDIS 3 refers to moderate infection, PEDIS 4 refers to severe infection) (Lipsky et al., 2016). Since RNA sequencing approach requires RNA with high quality and integrity, after RNA extraction from all of the clinical samples and initial assessment, 16 samples that had high-quality and integrity RNA were selected for RNA sequencing.

\section{Sample Collection}

After the DFI ulcer was cleaned with sterile $0.9 \% \mathrm{NaCl}$, a sterile single-use punch with a circular hollow blade was rotated around the affected area, and then the sharp debridement was collected by disposable forceps and preserved immediately in a $2 \mathrm{ml}$ RNAlater stabilization solution (Thermo Fisher Scientific,
Waltham, MA, United States) for $24 \mathrm{~h}$ at $4^{\circ} \mathrm{C}$ and then stored at $-80^{\circ} \mathrm{C}$ until processed.

\section{Sample Pretreatment}

Infected tissue specimens $(<25 \mathrm{mg})$ were homogenized in $1 \mathrm{ml}$ TRIzol reagent (Invitrogen, Carlsbad, CA, United States) using TissueRuptor II (Qiagen, Hilden, Germany) at $230 \mathrm{~V}$, $50 / 60 \mathrm{~Hz}$ for $10 \mathrm{~s}$.

\section{RNA Extraction}

The above pretreated tissue samples $(<25 \mathrm{mg})$ were incubated in $1 \mathrm{ml}$ TRIzol reagent for $5 \mathrm{~min}$ prior to further homogenization using 0.1 and $0.5 \mathrm{~mm}$ beads in a FastPrep24 instrument (MP Biomedicals, Irvine, CA, United States) with a velocity of $5 \mathrm{~m} / \mathrm{s}$ for 1 min while sitting on dry ice to break the bacterial cell wall. TRIzol ${ }^{\circledR}$ Plus RNA Purification Kit (Invitrogen, Carlsbad, CA, United States) was used to extract high-quality RNA from homogenized tissue samples. Extracted RNA was further treated with TURBO DNase (Invitrogen, Carlsbad, CA, United States) and purified by AMPure XP beads (Beckman Coulter Life Sciences, San Jose, CA, United States) according to the manufacturer's instructions.

\section{RNA Integrity, Synthesis of CDNA, and Illumina Sequencing}

Quality and integrity of extracted RNA were evaluated in an Agilent 2100 Bioanalyzer system using microfluidics-based electrophoresis on microfluidic chips (Agilent Technologies, Santa Clara, CA, United States) which produced an RNA integrity number (RIN) as an output. Illumina whole transcriptome library preparation with rRNA depletion was performed using the Illumina Ribo-Zero Gold Epidemiology kit (Illumina Inc., San Diego, CA, United States) on 16 selected high-quality RNA samples. The RNA sequencing was run on one Illumina NovaSeq 6000 S4 flow cell (300 Cycle) by the Australian Genome Research Facility to achieve $>150$ million pair-end reads of 150 nucleotides per sample.

\section{Processing of Metatranscriptome Data}

Sequencing reads were passed through the FastQC quality control pipeline Version 0.11.7 (Andrews, 2010) to visualize their quality. Sequencing reads were trimmed and quality-filtered with Trimmomatic tool version 0.39 (LEADING:5, TRAILING:5, SLIDINGWINDOW $=4: 15$, MINLEN $=50$ ) (Bolger et al., 2014). To filter human sequencing reads, trimmed reads were mapped to the human reference genome using ultrafast universal RNA-seq aligner (STAR v2.5.2a, human reference: GRCh37 genome including transcript annotation) (Dobin et al., 2013) and Burrows-Wheeler Alignment (BWA-mem version 0.7.15, human reference: GRCh38) (Li and Durbin, 2009). Putative non-human reads were then mapped to ribosome RNA databases (SILVA 16S, 23S, 28S, 18S rRNA, rfam 5S rRNA) using SortMeRNA v2.1 to identify and remove ribosomal RNA sequences (Kopylova et al., 2012). Putative bacterial mRNA was the target for further analyses (Figure 1). 


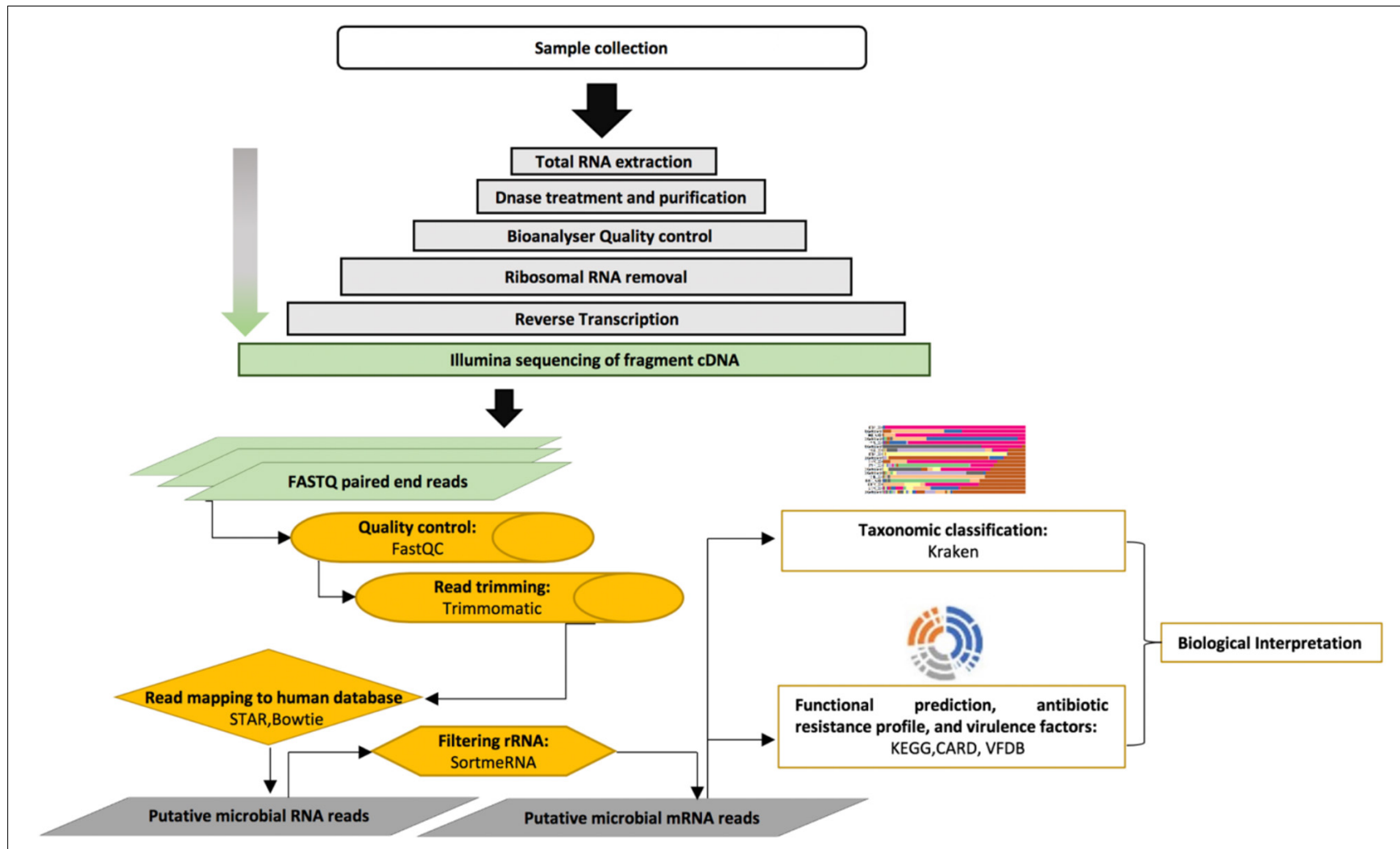

FIGURE 1 | Overview of the metatranscriptome pipeline applied in this study.

\section{Bacterial Taxonomic Classification, Functional Profiling, DFI Resistome, and Expressed Virulence Factors}

The taxonomic label assignment to sequencing reads was done using Kraken classifier v2 (an alignment program for assigning taxonomic labels to sequencing reads) (Wood and Salzberg, 2014). The database Kyoto Encyclopedia of Genes and Genomes (KEGG) (version 9 November 2016) was used to annotate the function of sequencing reads (Kanehisa and Goto, 2000) using DIAMOND v.0.99.

Visualization and statistical analysis were performed in Calypso 8.27 (Zakrzewski et al., 2016). ANOVA was used to determine if bacterial composition and function were significantly changed with infection severity.

The comprehensive antibiotic resistance database (CARD v3.0.1) (Jia et al., 2016) was used to identify bacterial transcripts carrying antibiotic resistance function (DFI resistome) using DIAMOND v.0.99. Antibiotic resistance genes in CARD were annotated as a present for a particular sample if at least $90 \%$ of the CARD reference sequence was covered by the metatranscriptome reads and with an average read fold of least 2.63.

The presence of bacterial virulence factors in metatranscriptome reads was evaluated using the Virulence Factor Database (VFDB) with at least $90 \%$ of coverage and an average read fold of 3.90 (Chen et al., 2016). The overview of the metatranscriptome pipeline used in this study is shown in Figure 1.

\section{RESULTS}

After the initial assessment of extracted RNA, 16 samples that had high integrity and quality RNA with either a mild $(25 \%)$, moderate $(31.25 \%)$, or severe infection $(43.75 \%)$ were investigated in this study. The average age of patients was $60.80 \pm 10.43$ years (range from 34 to 77 years). Three patients were female, and 13 patients were male. Three patients (2 females and one male) had Type 1 diabetes, while remaining patients suffered from Type 2 diabetes. All patients suffered from peripheral neuropathy (100\%) (Table 1).

\section{Microbial Community Composition}

RNAseq results in an average of 170 million paired-end reads (range 128 to 204 million) of 150 nucleotides per sample and on average 7.6 million paired-end reads per sample were taxonomically assigned to a bacterial taxon using Kraken. Fourteen bacterial phyla, 24 classes, 55 orders, 91 families, 109 genera, and 135 active species were identified in DFIs. Alignments of the sequencing reads using Kraken showed that the DFIs comprised of the phyla Proteobacteria, Firmicutes, Bacteroidetes, Fusobacteria, Actinobacteria, Tenericutes, Cyanobacteria, Spirochetes, Thermotogae, Acidobacteria, 
TABLE 1 | Demography of recruited patients in this study.

\begin{tabular}{|c|c|c|c|c|c|c|}
\hline $\begin{array}{l}\text { Patient } \\
\text { ID }\end{array}$ & Age & Gender & $\begin{array}{c}\text { Type of } \\
\text { diabetes } \\
(1,2)\end{array}$ & $\begin{array}{c}\text { Duration of } \\
\text { diabetes } \\
\text { (Year) }\end{array}$ & $\begin{array}{l}\begin{array}{c}\text { Duration } \\
\text { of ulcer } \\
\text { (weeks) }\end{array} \\
<2(0), 2-6 \\
\text { (1), >6 (2) }\end{array}$ & $\begin{array}{l}\text { PEDIS score } \\
2 \text { = Mild, } \\
\begin{array}{l}3 \text { = Moderate, } \\
4 \text { = Severe }\end{array}\end{array}$ \\
\hline DFI109 & 51 & Female & 1 & 30 & 2 & 2 \\
\hline DFI111 & 51 & Female & 1 & 30 & 2 & 3 \\
\hline DFI112 & 68 & Male & 2 & 12 & 2 & 2 \\
\hline DFI113 & 54 & Male & 1 & 23 & 2 & 3 \\
\hline DFI114 & 72 & Male & 2 & 20 & 1 & 2 \\
\hline DFI117 & 34 & Male & 2 & 14 & 2 & 3 \\
\hline DFI119 & 61 & Male & 2 & 13 & 1 & 2 \\
\hline DFI121 & 61 & Female & 2 & 12 & 2 & 3 \\
\hline DFI126 & 54 & Male & 2 & 11 & 2 & 3 \\
\hline DFI153 & 65 & Male & 2 & 20 & 2 & 4 \\
\hline DFI155 & 62 & Male & 2 & 21 & 1 & 4 \\
\hline DFI160 & 59 & Male & 2 & 2 & 1 & 4 \\
\hline DFI161 & 68 & Male & 2 & 19 & 0 & 4 \\
\hline DFI166 & 71 & Male & 2 & 22 & 2 & 4 \\
\hline DFI167 & 77 & Male & 2 & 35 & 2 & 4 \\
\hline DFI171 & 64 & Male & 2 & 17 & 1 & 4 \\
\hline
\end{tabular}

Planctomycetes, Verrucomicrobia, Aquificae, and Deferribacteres in descending order of the mean abundance in the samples. Proteobacteria, Firmicutes, and Bacteroidetes constituted the most abundant phyla. The abundance of individual taxa is visualized in Figure 2.

The genera Proteus, Porphyromonas, Anaerococcus, Parvimonas, and Peptoniphilus constituted the highest number of assigned sequencing reads in descending order. The genera Mycoplasma, Spiroplasma, and Vibrio were significantly abundant in moderate infections $(P<0.05$, Figure 3$)$.

Regardless of infection severity, Proteus mirabilis, Porphyromonas asaccharolytica, Parvimonas micra, Anaerococcus mediterraneensis, and Peptoniphilus harei had the highest number of assigned transcripts (Figure 4). Among samples with mild and moderate infections $(N=9)$, three samples were dominated by Staphylococcus aureus. Porphyromonas asaccharolytica was detected in all severe samples $(n=7)$ and dominant in four samples.

\section{Functional Annotations of the Transcripts in DFIs}

Using similarity searches to the KEGG database, 132 functional pathways were identified in the DFIs. Six pathways were significantly changed in different infection severities $(P<0.05)$ (Table 2).

Pathways involved in ribosome and thiamine metabolism were the most abundant pathways (Figure 5A). A high number of ribosomal transcripts, particularly in S. aureus, $P$. asaccharolytica, and Finegoldia magna, may indicate high transcriptional activity and important metabolic roles of these species (Figure 5B).
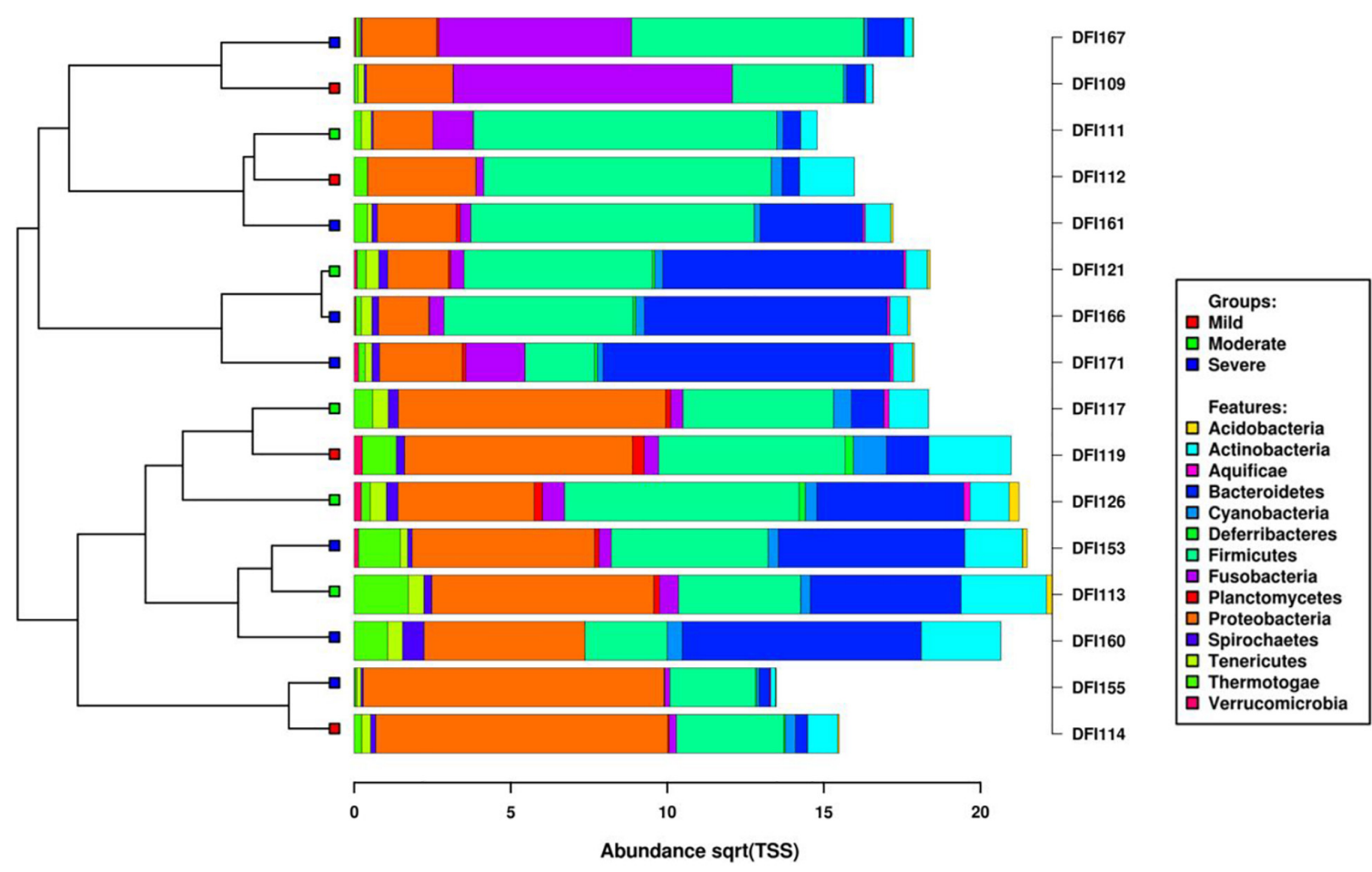

FIGURE 2 | Clustered bar chart based on hierarchical clustering of the Bray-Curtis distances of the DFIs based on the taxonomic composition of active bacterial phyla as determined by metatranscriptome sequencing predicted using Kraken and visualized in Calypso. The horizontal axis is the square root abundances of the identified taxa. 

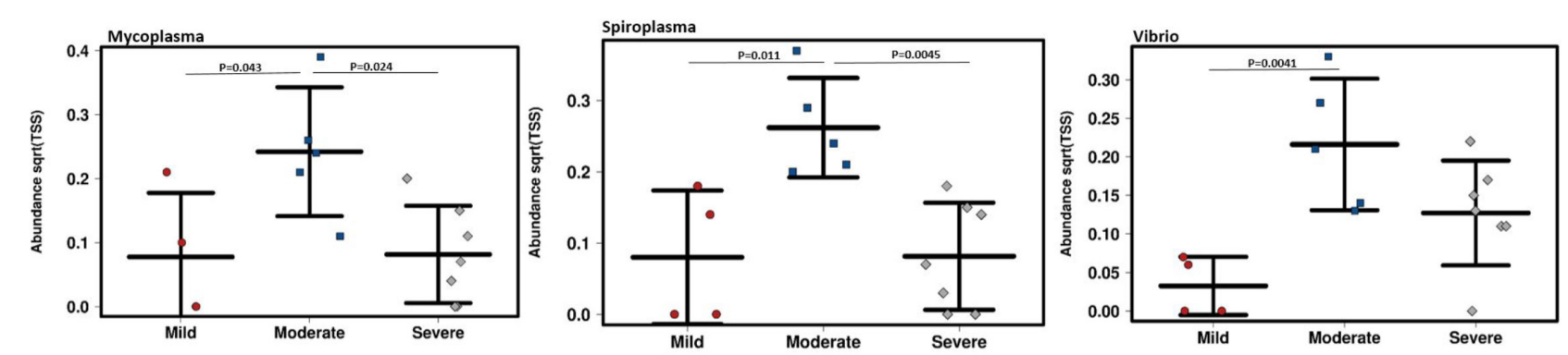

FIGURE 3 | The genera Mycoplasma, Spiroplasma, and Vibrio significantly increased in moderate infections $(P<0.05)$. The vertical axis shows the square root abundances of each bacterial genus.

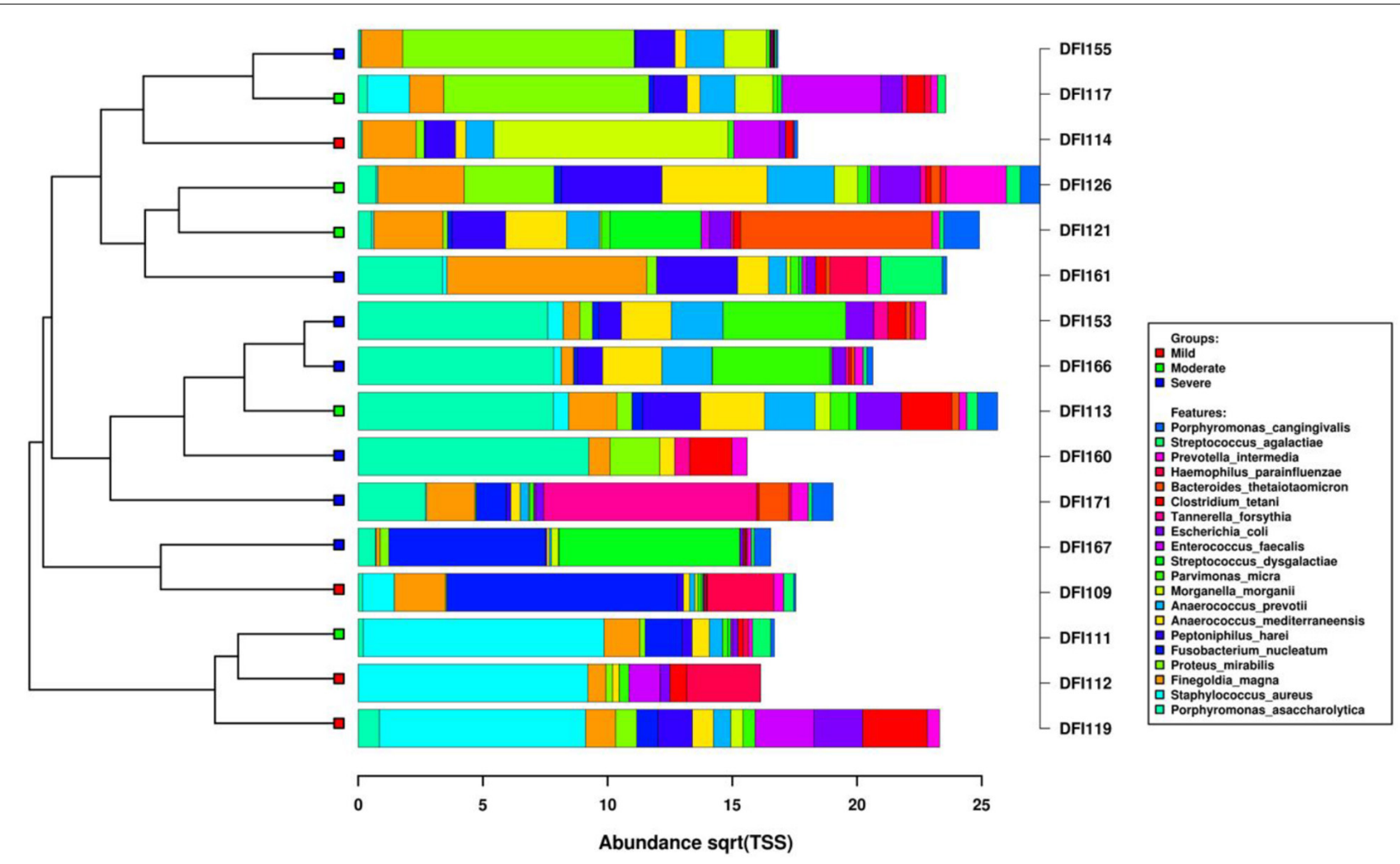

FIGURE 4 | Clustered bar chart of the top 20 active bacterial species. The dendrogram was calculated using the hierarchical clustering of Bray-Curtis distances including the top 20 species assigned using Kraken. The horizontal axis is the square root abundance of the identified taxa.

\section{DFI Resistome Prediction}

Assignment of bacterial transcripts to the CARD database revealed the presence of 131 different genes directly or indirectly involved in antibiotic resistance mechanisms using the cut-off of $>90 \%$ reference sequence coverage with the average read fold of at least 2.6 in 12 samples. No CARD feature was identified in four samples. The DFI resistome was mainly comprised of genes involved in the multidrug efflux pumps/exporters, resistance to beta-lactam, macrolide, and tetracycline antibiotics. TEM betalactamase resistance genes and genes involved in multidrug efflux pumps/exporters were detected in 11 samples. Tetracycline resistance gene (tet) and Erm methyltransferase were also detected in six and four samples, respectively. Furthermore, Gene $c f x A$, encoding a class A beta-lactamase was detected in five samples (Figure 6).

\section{Expressed Bacterial Virulence Factors in DFIs}

The assignment of bacterial transcripts to the VFDB revealed the presence of 225 different mechanisms involved in bacterial pathogenicity using $90 \%$ coverage with the average read fold of at least 3.90 in seven samples. No VFDB feature was identified in nine samples. Overall, the most common virulence factors identified in DFIs comprised pathways involved in synthesizing 
TABLE 2 | Pathways significantly changed between infection severities $(P<0.05)$.

\begin{tabular}{|c|c|c|}
\hline Pathway & Class & $\begin{array}{l}\text { Increased in } \\
\text { infection } \\
\text { severity } \\
\text { (PEDIS) }\end{array}$ \\
\hline Lipoic acid metabolism & $\begin{array}{l}\text { Metabolism of vitamins and } \\
\text { cofactors }\end{array}$ & Mild \\
\hline Two-component systems & Signal transduction & Mild \\
\hline $\begin{array}{l}\text { Bacterial invasion to epithelial } \\
\text { cells }\end{array}$ & Bacterial infectious disease & Mild \\
\hline Glycerolipid metabolism & Metabolism of lipid & Moderate \\
\hline TCA cycle & Metabolism of carbohydrate & Moderate \\
\hline Mismatch repair & Replication and repairing DNA & Severe \\
\hline
\end{tabular}

and regulation of siderophores (iron-chelating molecules). The next common virulence factors were involved in bacterial cellsurface components (fimbria and flagellum) which facilitate adhesion, colonization, and movement of bacterial cells. Other pathways involved in the pathogenicity of pathogens are shown in Figure 7.

\section{DISCUSSION}

Diabetic foot infections are the most severe and costly complication of diabetes. With regards to the diabetic population, $50 \%$ of them are recognized to have ulcerated feet (Gupta and Kumar, 2019). Progression of DFIs to more complicated scenarios, such as minor and major amputation of a lower limb occurs every $30 \mathrm{~s}$ in the world which can influence the quality of life in many diabetic people worldwide ${ }^{1}$.

Identification of pathogenic bacteria is the first essential step to monitor and control the etiology of DFIs accurately (Sadeghpour Heravi et al., 2019). However, a large portion of bacterial species are not culturable using traditional methods (Suryaletha et al., 2018). While many human disorders have been linked to a shift in the bacterial composition/function (Nowicki et al., 2018), it is still unclear how this fluctuation may influence the development of DFIs.

Metatranscriptomics provides an extraordinary opportunity to systematically study bacterial communities (Zhao et al., 2016) including information about active bacterial compositions/functions which are necessary for the initiation and progression of the infection.

This study provides insights into the transcriptionally active bacterial population and its functionality in DFIs by means of a metatranscriptomic approach.

In this study, a high abundance of the transcripts assigned to the genera Proteus, Porphyromonas, Anaerococcus, Parvimonas, Peptoniphilus, Prevotella, Fingoldia, and Streptococcus were identified in DFIs. Taxonomic annotation revealed that bacterial pathogens in mild infection (PEDIS 2) were mainly

${ }^{1} \mathrm{Https} / / /$ Idf.Org/Our-Activities/Care-Prevention/Diabetic-Foot.Html/ Accessed:2020
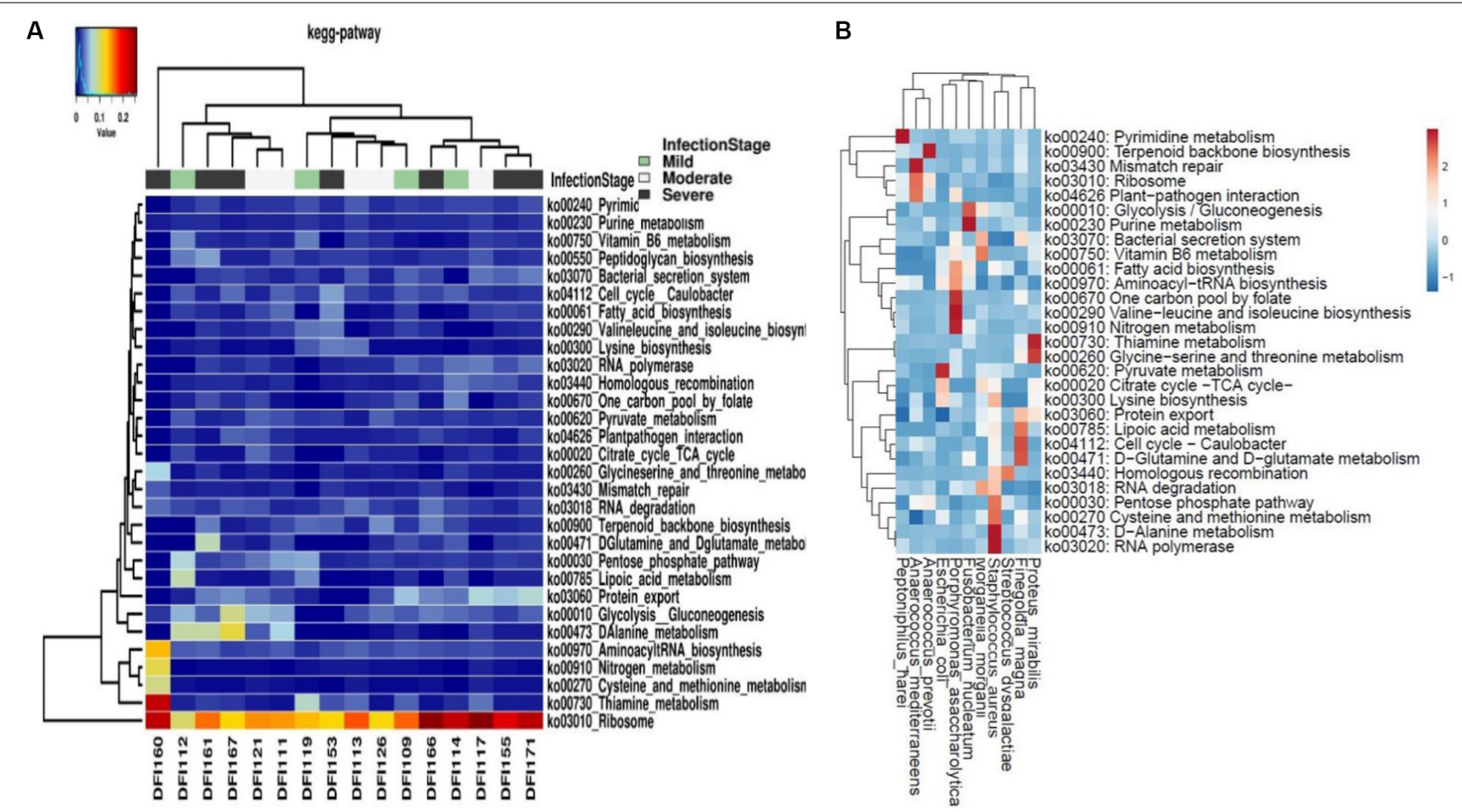

FIGURE 5 | (A) Top 30 metabolic pathways predicted using the KEGG and visualized in Calypso. (B) Heatmap of KEGG pathways in highly transcriptionally active bacteria. 

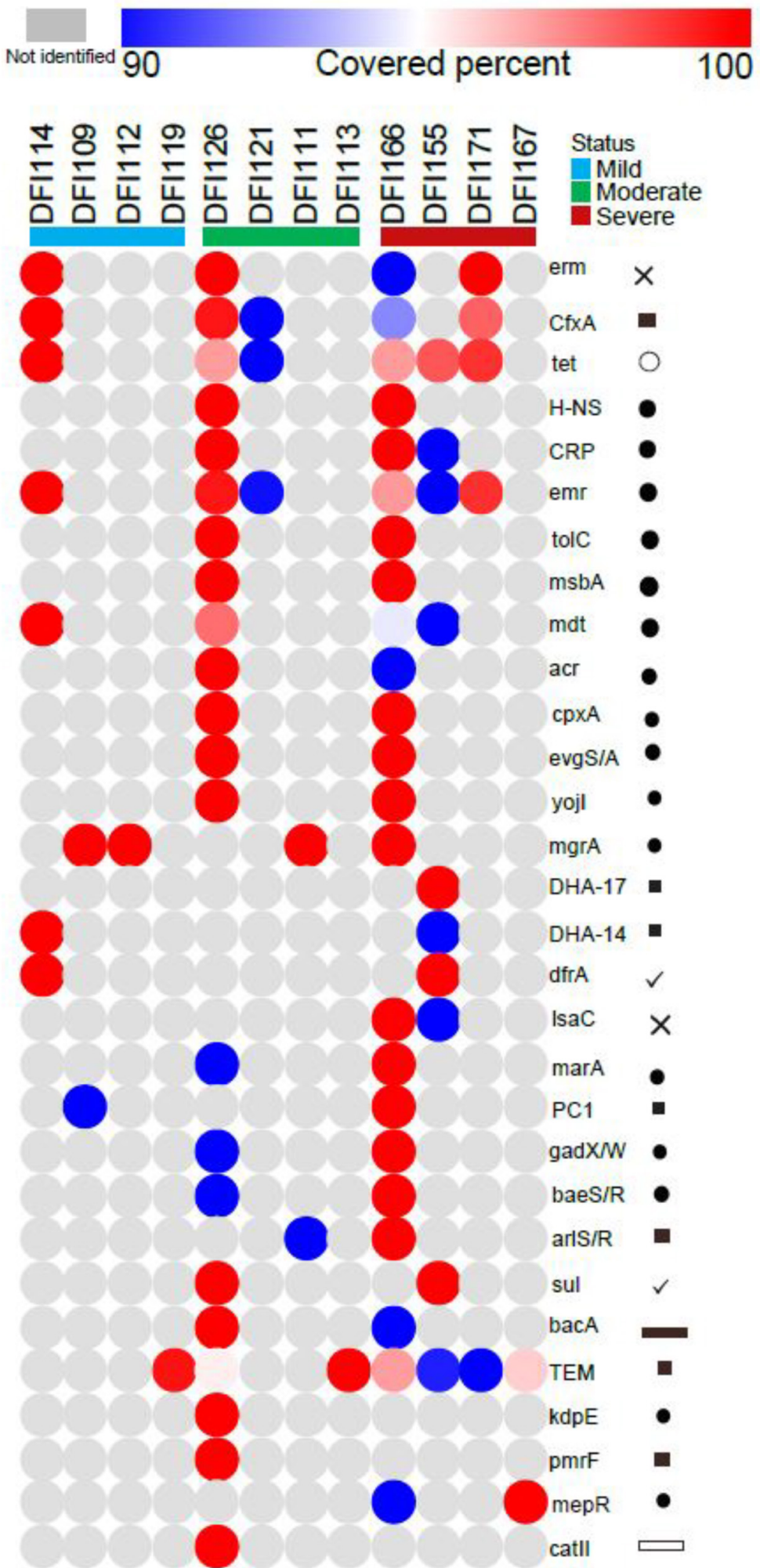

FIGURE 6 | Detected antibiotic resistome in 12 DFI samples using the CARD. Antibiotic resistance genes encoding beta-lactam, macrolide, and tetracycline antibiotics were annotated mostly in moderate and severe infection. Genes related to multidrug efflux pumps/exporters and beta-lactam antibiotics were detected in 11 samples ( $\times$ : macrolides, $\mathbf{\square}$ : $\beta$-lactam, o: polyketides, $\bullet$ : multidrug efflux pumps/exporters, $\checkmark$ : sulfonamide, trimethoprim, $\mathbf{\square}$ : bacitracin, $\mathbf{\square}$ chloramphenicol). Circle color indicates the percentage coverage of the reference CARD sequence by the RNA-seq data (red/blue) or the absence in a sample (gray). Similar genes with similar functions were considered as one group. For instance, TEM-1, TEM-116, TEM-198, and TEM-157 were grouped as TEM. 


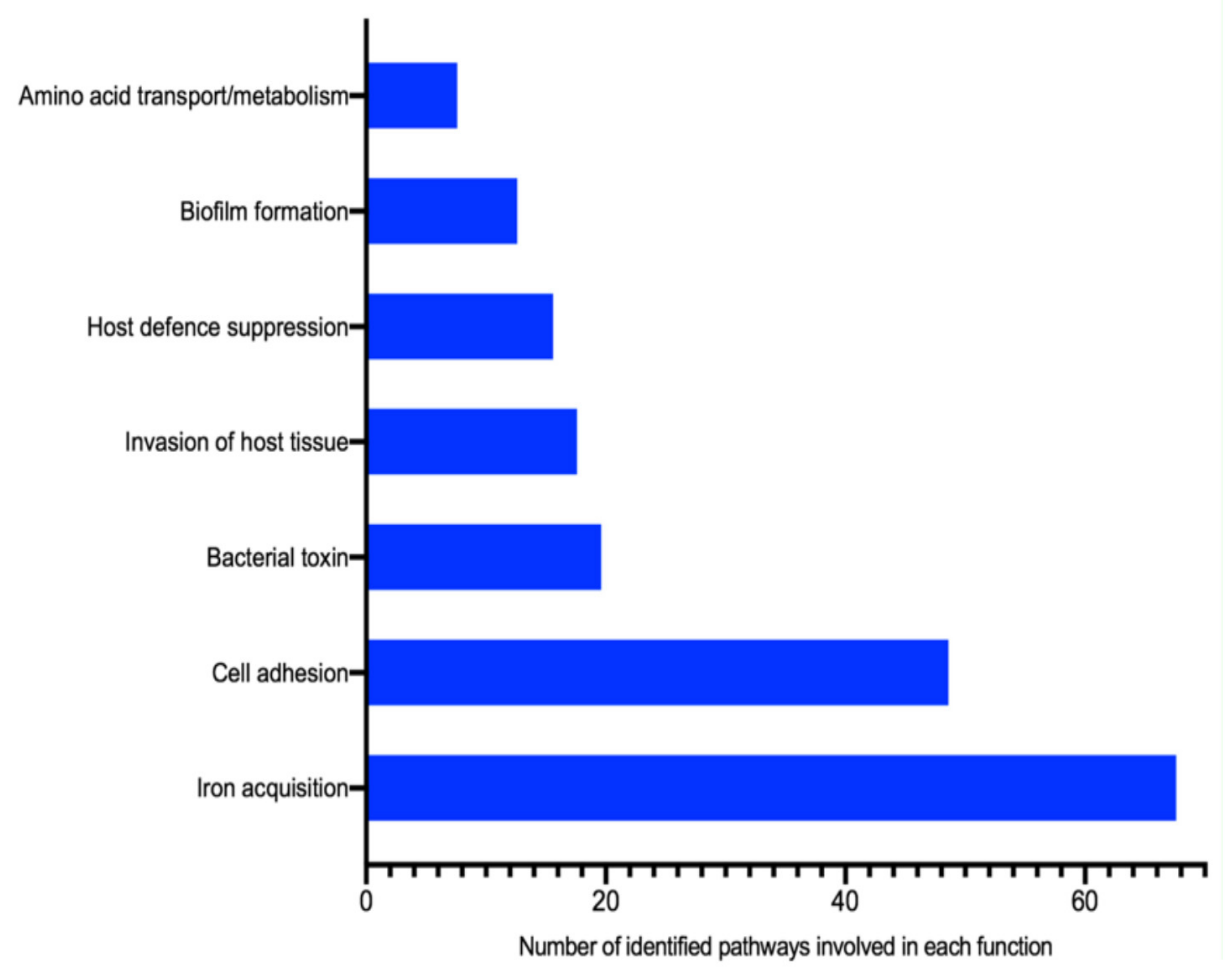

FIGURE 7 | Identified bacterial virulence factors in DFIs using the VFDB.

S. aureus, F. magna, Fusobacterium nucleatum, and Proteus mirabilis which were commonly identified in DFIs (Gardiner et al., 2017). In moderate infections (PEDIS 3), F. magna, $P$. harei, Morganella morganii, and Anaerococcus prevotii were the most common assigned transcripts. In severe infections (PEDIS 4) P. asaccharolytica, F. magna, Parvimonas mica, Anaerococcus mediterraneensis, and Proteus mirabilis were predominant (Figure 4). These findings suggested that aerobic Gram-positive cocci such as $S$. aureus which has been the most described pathogen in DFIs were less common as the severity of infection increased. Transcripts assigned to the genera Spiroplasma, Vibrio, and Mycoplasma were significantly increased in moderate infections $(P<0.05)$, which indicated a major metabolic role of these genera in DFIs. The presence of transcripts assigned to both metabolically active aerobic (such as $S$. aureus, Proteus mirabilis, and Escherichia coli) and anaerobic microorganisms (such as $P$. asaccharolytica, F. magna, and Fusobacterium nucleatum) indicated the dual status of the bacterial lifestyle and the complexity of the bacterial communities in DFIs. The high prevalence of anaerobes and fastidious microorganisms (such as Anaerococcus spp. and Peptoniphilus spp.) may explain the inadequacy of culture-based methods in isolating the entire pathogens in DFIs.

Transcripts assigned to thiamin pathways dominated the DFIs. Since this pathway is a vital pathway in bacterial pathogenesis (Du et al., 2011; Costliow and Degnan, 2017) disrupting this pathway may lead to bacterial cell death and may suggest a novel target for treatment of DFIs (Schauer et al., 2009; Monjas et al., 2016). Also, the great abundance of ribosomal transcripts indicated the high translational activity of the bacterial population in our study (Bisanz et al., 2014) which may suggest the use and development of antibiotics that specifically target ribosomal function and subunits in bacterial pathogens.

The high abundance of bacterial transcripts assigned to a two-component system (TCS) in the mild stages of infection in this study $(P<0.05)$ (Table 2$)$ and the importance of the TCS in bacterial survival may suggest a key role of the system as a new antimicrobial target. Furthermore, the high abundance of transcripts assigned to the bacterial pathway "invasion of epithelial cells" during mild infections $(P<0.05)$ may also suggest invading ability of pathogenic bacteria to enter epithelial cells and expand the infection in the early stages of infection (Ribet and Cossart et al., 2015; Table 2).

We have identified that most DFIs have complex metabolically active bacterial communities, thus understanding which bacteria contribute to the infective process could help to reduce the overuse of commonly prescribed antibiotics. Beta-lactam antibiotics are the most broadly prescribed antibiotics in the empirical therapy of DFIs. Extended-spectrum $\beta$-lactamase (ESBL) is the main resistance mechanism in Gram-negative bacteria, which results in multidrug resistance (MDR) pathogens. Based on our findings, genes conferring resistance to betalactams and genes involved in multidrug efflux pumps/exporters 
were detected in eleven samples. Tetracycline resistance genes (tet) and Erm methyltransferases were detected in six samples (Figure 6). Alarming levels of ESBL phenotypes, which were resistant to many classes of commonly prescribed antibiotics such as penicillins and cephalosporins have been reported in many different studies which were concordant with our findings suggesting more precaution in the prescription of these antibiotics in the treatment of DFIs (Motta et al., 2003; Turhan et al., 2013).

Also, the iron acquisition system (synthesize and regulation of siderophores) was the most common mechanism involved in the pathogenicity of bacterial cells in DFIs. Pathways involved in the synthesis and regulation of cell-surface components associated with adhesion, colonization, and movement of bacterial cells were the next common virulence factors. These virulence factors may help bacteria compete for scares resources and survive the host wound proteases. This may explain the importance of the aforementioned systems in the pathogenicity of bacterial cells and targeting these factors to prevent bacterial cells more effectively (Figure 7).

However, obtaining RNA with high RIN from infected clinical samples suitable for RNA sequencing approach is very challenging, after the initial assessment of the extracted RNA, we were limited to low sample size as RNA in some infected tissue may have already been degraded in situ.

RNA sequencing is very costly as well and needs deep sequencing (average of 170 million reads per sample in this study) to be able to capture enough bacterial RNA signals as $>95 \%$ of sequencing reads belonging to human RNA reads in clinical samples. However, recent advances in the genomics field over the past quarter-century have led to considerable reductions in the sequencing costs. Since these methods are becoming frontlines in medical laboratories, it may be projected that this reduction to be continued in order to influence the scale and scope of research projects investigating genomic aspects of bacterial communities (Marshall et al., 2017).

To the best of our knowledge, this is the first study to apply an RNA-sequencing technique to profile the active bacterial communities in DFIs. Our findings can help to identify the composition and function of bacterial communities in DFIs. However, further experimental research is needed to evaluate the pathogenicity of the identified bacterial species and the application of detected pathways in the treatment of DFIs.

\section{CONCLUSION}

Based on our findings, treatment strategies targeting a single species or specific bacterial pathways might be ineffective

\section{REFERENCES}

Andrews, S. (2010). "FastQC: a quality control tool for high throughput sequence data," in Babraham Bioinformatics, (Cambridge: Babraham Institute). in the treatment of DFIs, and a multifaceted therapy is required. Abundant pathogens and pathways identified in this study may be possible biomarkers to prevent infection in the future.

\section{DATA AVAILABILITY STATEMENT}

The datasets presented in this study can be found in online repositories. The names of the repository/repositories and accession number(s) can be found at: https://www.ncbi.nlm.nih. gov/, PRJNA563930.

\section{ETHICS STATEMENT}

This study was approved by the South West Sydney Local Health District Research and Ethics Committee (HREC/14/LPOOL/487, SSA/14/LPOOL/489) and Macquarie University Human Ethics Committee (Reference No. 5201500839). The patients/participants provided their written informed consent to participate in this study.

\section{AUTHOR CONTRIBUTIONS}

FH conducted the laboratory experiments and data analysis and wrote the manuscript. MZ analyzed the data, and reviewed and commented on the manuscript. KV and MM reviewed and commented on the manuscript. $\mathrm{HH}$ designed the project, monitored the laboratory experiments, and reviewed and commented on the manuscript. All the authors read the final manuscript and consented the publication.

\section{FUNDING}

This project was supported by Macquarie Research Development Grant Scheme (Project No. 9201601601) from Macquarie University, Sydney, NSW, Australia. FH was in receipt of an Australian International Research Training Program (iRTP) Scholarship.

\section{ACKNOWLEDGMENTS}

A special thanks to Dr. Hamidreza Aboulkheyr Estarabadi for his valuable advice and helping in the preparation of Figure 6.

Bisanz, J. E., Macklaim, J. M., Gloor, G. B., and Reid, G. (2014). Bacterial metatranscriptome analysis of a probiotic yogurt using an RNA-Seq approach. Int. Dairy J. 39, 284-292. doi: 10.1016/j.idairyj.2014. 07.010 
Bolger, A. M., Lohse, M., and Usadel, B. (2014). Trimmomatic: a flexible trimmer for Illumina sequence data. Bioinformatics 30, 2114-2120. doi: 10.1093/ bioinformatics/btu170

Chen, L., Zheng, D., Liu, B., Yang, J., and Jin, Q. (2016). VFDB 2016: hierarchical and refined dataset for big data analysis-10 years on. Nucleic Acids Res. 44, D694-D697.

Costliow, Z. A., and Degnan, P. H. (2017). Thiamine acquisition strategies impact metabolism and competition in the gut microbe Bacteroides thetaiotaomicron. MSystems 2:e0116-17.

Dobin, A., Davis, C. A., Schlesinger, F., Drenkow, J., Zaleski, C., Jha, S., et al. (2013). STAR: ultrafast universal RNA-seq aligner. Bioinformatics 29, 15-21. doi: 10.1093/bioinformatics/bts635

Du, Q., Wang, H., and Xie, J. (2011). Thiamin (vitamin B1) biosynthesis and regulation: a rich source of antimicrobial drug targets? In. J. Biol. Sci. 7, 41-52. doi: 10.7150/ijbs.7.41

Gardiner, M., Vicaretti, M., Sparks, J., Bansal, S., Bush, S., Liu, M., et al. (2017). A longitudinal study of the diabetic skin and wound microbiome. PeerJ 5:e3543. doi: $10.7717 /$ peerj.3543

Gupta, A., and Kumar, V. (2019). Multidisciplinary approach to prevent limb amputation in diabetic patients. J. Endocrinol. Metab. 9, 120-126. doi: 10. 14740/jem615

Heravi, F. S., Zakrzewski, M., Vickery, K., and Hu, H. (2020). Host DNA depletion efficiency of microbiome DNA enrichment methods in infected tissue samples. J. Microbiol. Methods 170:105856. doi: 10.1016/j.mimet.2020.105856

Jia, B., Raphenya, A. R., Alcock, B., Waglechner, N., Guo, P., Tsang, K. K., et al. (2016). CARD 2017: expansion and model-centric curation of the comprehensive antibiotic resistance database. Nucleic Acids Res. 45, D566D573.

Kanehisa, M., and Goto, S. (2000). KEGG: kyoto encyclopedia of genes and genomes. Nucleic Acids Res. 28, 27-30.

Kopylova, E., Noé, L., and Touzet, H. (2012). SortMeRNA: fast and accurate filtering of ribosomal RNAs in metatranscriptomic data. Bioinformatics 28, 3211-3217. doi: 10.1093/bioinformatics/bts611

Lavery, L. A., Armstrong, D. G., Wunderlich, R. P., Tredwell, J., and Boulton, A. J. (2003). Diabetic foot syndrome: evaluating the prevalence and incidence of foot pathology in Mexican Americans and non-Hispanic whites from a diabetes disease management cohort. Diabetes Care 26, 1435-1438. doi: 10. 2337/diacare.26.5.1435

Li, H., and Durbin, R. (2009). Fast and accurate short read alignment with Burrows-Wheeler transform. Bioinformatics 25, 1754-1760. doi: 10.1093/ bioinformatics/btp324

Lipsky, B. A., Aragón-Sánchez, J., Diggle, M., Embil, J., Kono, S., Lavery, L., et al. (2016). IWGDF guidance on the diagnosis and management of foot infections in persons with diabetes. Diabetes Metab. Res. Rev. 32, 45-74. doi: 10.1002/ dmrr.2699

Marshall, D. A., Macdonald, K. V., Robinson, J. O., Barcellos, L. F., Gianfrancesco, M., Helm, M., et al. (2017). The price of whole-genome sequencing may be decreasing, but who will be sequenced? Pers. Med. 14, 203-211. doi: 10.2217/ pme-2016-0075

Monjas, L., Swier, L., De Voogd, A., Oudshoorn, R., Hirsch, A., and Slotboom, D. (2016). Design and synthesis of thiamine analogues to study their binding to the ECF transporter for thiamine in bacteria. MedChemComm 7, 966-971. doi: $10.1039 / \mathrm{c} 6 \mathrm{md} 00022 \mathrm{c}$

Motta, R., Oliveira, M., Magalhaes, P., Dias, A., Aragao, L., Forti, A., et al. (2003). Plasmid-mediated extended-spectrum beta-lactamase-producing strains of Enterobacteriaceae isolated from diabetes foot infections in a Brazilian diabetic center. Braz. J. Infect. Dis. 7, 129-134. doi: 10.1590/s1413-867020030002 00006

Nowicki, E. M., Shroff, R., Singleton, J. A., Renaud, D. E., Wallace, D., Drury, J., et al. (2018). Microbiota and metatranscriptome changes accompanying the onset of gingivitis. mBio 9, e00575-18.

Ribet, D., and Cossart, P. (2015). How bacterial pathogens colonize their hosts and invade deeper tissues. Microb. Infect. 17, 173-183. doi: 10.1016/j.micinf.2015. 01.004

Sadeghpour Heravi, F., Zakrzewski, M., Vickery, K., Armstrong, G. D., and Hu, H. (2019). Bacterial diversity of diabetic foot ulcers: current status and future prospectives. J. Clin. Med. 8:E1935.

Schauer, K., Stolz, J., Scherer, S., and Fuchs, T. M. (2009). Both thiamine uptake and biosynthesis of thiamine precursors are required for intracellular replication of Listeria monocytogenes. J. Bacteriol. 191, 2218-2227. doi: 10.1128/jb.01636-08 Smith, K., Collier, A., Townsend, E. M., O’donnell, L. E., Bal, A. M., Butcher, J., et al. (2016). One step closer to understanding the role of bacteria in diabetic foot ulcers: characterising the microbiome of ulcers. BMC Microbiol. 16:54. doi: 10.1186/s12866-016-0665-z

Suryaletha, K., John, J., Radhakrishnan, M. P., George, S., and Thomas, S. (2018). Metataxonomic approach to decipher the polymicrobial burden in diabetic foot ulcer and its biofilm mode of infection. Int. Wound J. 15, 473-481. doi: 10.1111/iwj.12888

Turhan, V., Mutluoglu, M., Acar, A., Hatipoglu, M., Onem, Y., Uzun, G., et al. (2013). Increasing incidence of Gram-negative organisms in bacterial agents isolated from diabetic foot ulcers. J. Infect. Dev. Ctries 7, 707-712. doi: 10.3855/ jidc. 2967

Wood, D. E., and Salzberg, S. L. (2014). Kraken: ultrafast metagenomic sequence classification using exact alignments. Genome Biol. 15:R46.

Zakrzewski, M., Proietti, C., Ellis, J. J., Hasan, S., Brion, M.-J., Berger, B., et al. (2016). Calypso: a user-friendly web-server for mining and visualizing microbiome-environment interactions. Bioinformatics 33, 782-783.

Zhao, S., Zhang, B., Zhang, Y., Gordon, W., Du, S., Paradis, T., et al. (2016). "Bioinformatics for RNA-Seq data analysis," in Bioinformatics-Updated Features and Applications, (London: InTech), 125-149.

Conflict of Interest: The authors declare that the research was conducted in the absence of any commercial or financial relationships that could be construed as a potential conflict of interest.

Copyright (c) 2020 Heravi, Zakrzewski, Vickery, Malone and Hu. This is an openaccess article distributed under the terms of the Creative Commons Attribution License (CC BY). The use, distribution or reproduction in other forums is permitted, provided the original author(s) and the copyright owner(s) are credited and that the original publication in this journal is cited, in accordance with accepted academic practice. No use, distribution or reproduction is permitted which does not comply with these terms. 